DSF preprint $96 / 42$

\title{
QUANTIZED MAXWELL THEORY IN A CONFORMALLY INVARIANT GAUGE
}

Giampiero Esposito

\author{
Istituto Nazionale di Fisica Nucleare, Sezione di Napoli \\ Mostra d'Oltremare Padiglione 20, 80125 Napoli, Italy; \\ Dipartimento di Scienze Fisiche \\ Mostra d'Oltremare Padiglione 19, 80125 Napoli, Italy. \\ e-mail: esposito@napoli.infn.it
}

\begin{abstract}
Maxwell theory can be studied in a gauge which is invariant under conformal rescalings of the metric, and first proposed by Eastwood and Singer. This paper studies the corresponding quantization in flat Euclidean 4-space. The resulting ghost operator is a fourth-order elliptic operator, while the operator $\mathcal{P}$ on perturbations $A_{\mu}$ of the potential is a sixth-order elliptic operator. The operator $\mathcal{P}$ may be reduced to a second-order nonminimal operator if a dimensionless gauge parameter tends to infinity. Gauge-invariant boundary conditions are obtained by setting to zero at the boundary the whole set of $A_{\mu}$ perturbations, jointly with ghost perturbations and their normal derivative. This is made possible by the fourth-order nature of the ghost operator. An analytic representation of the ghost basis functions is also obtained.
\end{abstract}




\section{Quantized Maxwell Theory in a Conformally Invariant Gauge}

The recent attempts to quantize Euclidean Maxwell theory in quantum cosmological backgrounds have led to a detailed investigation of the quantized Maxwell field in covariant and non-covariant gauges on manifolds with boundary [1-7]. The main emphasis has been on the use of analytic or geometric techniques to evaluate the one-loop semiclassical approximation of the wave function of the universe, when magnetic or electric boundary conditions are imposed. In the former case one sets to zero at the boundary the tangential components $A_{k}$ of the potential (the background value of $A_{\mu}$ is taken to vanish), the realvalued ghost fields $\omega$ and $\psi$ (or, equivalently, a complex-valued ghost 0 -form $\varepsilon$ ), and the gauge-averaging functional $\Phi(A)$ :

$$
\begin{gathered}
{\left[A_{k}\right]_{\partial M}=0,} \\
{[\varepsilon]_{\partial M}=0} \\
{[\Phi(A)]_{\partial M}=0 .}
\end{gathered}
$$

In the electric scheme one sets instead to zero at the boundary the normal component of $A_{\mu}$, jointly with the normal derivative of the ghost and the normal derivative of $A_{k}$ :

$$
\begin{gathered}
{\left[A_{0}\right]_{\partial M}=0} \\
{[\partial \varepsilon / \partial n]_{\partial M}=0,} \\
{\left[\partial A_{k} / \partial n\right]_{\partial M}=0 .}
\end{gathered}
$$

One may check that the boundary conditions (1)-(3) and (4)-(6) are invariant under infinitesimal gauge transformations on $A_{\mu}$, as well as under BRST transformations [5].

On the other hand, the gauge-averaging functionals studied in Refs. [1-7] were not conformally invariant, although a conformally invariant choice of gauge was already known, at the classical level, from the work of Ref. [8]. It has been therefore our aim to investigate the quantum counterpart of the conformally invariant scheme proposed in Ref. [8], to complete the current work on quantized gauge fields. For this purpose, we have studied a portion of flat Euclidean 4-space bounded by 3-dimensional surfaces. The vanishing 
curvature of the 4-dimensional background is helpful to obtain a preliminary understanding of the quantum operators, which will be shown to have highly non-trivial properties. In our scheme, all curvature effects result from the boundary only.

In flat Euclidean 4-space, the conformally invariant gauge proposed in Ref. [8] reads (hereafter $b, c=0,1,2,3$ )

$$
\nabla_{b} \nabla^{b} \nabla^{c} A_{c}=\square \nabla^{c} A_{c}=0
$$

If the classical potential is subject to an infinitesimal gauge transformation

$$
{ }^{f} A_{b}=A_{b}+\nabla_{b} f
$$

the gauge condition (7) is satisfied by ${ }^{f} A_{b}$ if and only if $f$ obeys the fourth-order equation

$$
\square^{2} f=0
$$

where $\square^{2}$ is the $\square$ operator composed with itself, i.e. $\square^{2} \equiv g^{a b} g^{c d} \nabla_{a} \nabla_{b} \nabla_{c} \nabla_{d}$.

In the quantum theory via path integrals, however, one performs Gaussian averages over gauge functionals $\Phi(A)$ which ensure that well-defined Feynman Green's functions for the $\mathcal{P}$ operator on $A_{b}$, and for the ghost operator, actually exist [5,9]. This means that the left-hand side of Eq. (7) is no longer set to zero. One defines instead a gauge-averaging functional

$$
\Phi(A) \equiv \square \nabla^{b} A_{b}
$$

and the gauge-averaging term $\frac{1}{2 \alpha}[\Phi(A)]^{2}$, with $\alpha$ a dimensionless parameter, is added to the Maxwell Lagrangian $\frac{1}{4} F_{a b} F^{a b}$. A double integration by parts is then necessary to express the Euclidean Lagrangian in the form $\frac{1}{2} A_{b} \mathcal{P}^{b c} A_{c}$, where

$$
\mathcal{P}^{b c} \equiv-g^{b c} \square+\left(1-\frac{1}{\alpha} \square^{2}\right) \nabla^{b} \nabla^{c}
$$

The operator $\mathcal{P}^{b c}$ is a complicated sixth-order elliptic operator, and it is unclear how to deal properly with it for finite values of $\alpha$. However, in the limit as $\alpha \rightarrow \infty$, it reduces to the following second-order operator:

$$
P^{b c}=-g^{b c} \square+\nabla^{b} \nabla^{c}
$$


This operator remains non-minimal, since the term $\nabla^{b} \nabla^{c}$ survives. In this particular case, we still need to specify boundary conditions on $A_{b}$ and ghost perturbations. For this purpose, we put to zero at the boundary the whole set of $A_{b}$ perturbations:

$$
\left[A_{b}\right]_{\partial M}=0 \forall b=0,1,2,3,
$$

and we require invariance of (13) under infinitesimal gauge transformations on $A_{b}$. This leads to (hereafter $\tau$ is a radial coordinate [1-4])

$$
\begin{gathered}
{[\varepsilon]_{\partial M}=0} \\
{[\partial \varepsilon / \partial \tau]_{\partial M}=0 .}
\end{gathered}
$$

Condition (14) results from the gauge invariance of the Dirichlet condition on $A_{k}, \forall k=$ $1,2,3$, and condition (15) results from the gauge invariance of the Dirichlet condition on $A_{0}$. Note that it would be inconsistent to impose the boundary conditions (13)-(15) when the Lorentz gauge-averaging functional is chosen, since the corresponding ghost operator is second-order.

When two boundary 3 -surfaces occur, (14) and (15) lead to

$$
\begin{gathered}
{[\varepsilon]_{\Sigma_{1}}=[\varepsilon]_{\Sigma_{2}}=0,} \\
{[\partial \varepsilon / \partial \tau]_{\Sigma_{1}}=[\partial \varepsilon / \partial \tau]_{\Sigma_{2}}=0 .}
\end{gathered}
$$

When Eq. (10) is used, and the ghost operator is hence $\square^{2}$, the four boundary conditions (16) and (17) provide enough conditions to determine completely the coefficients $C_{1}, \ldots, C_{4}$ in the linear combination

$$
\varepsilon_{(\lambda)}=\sum_{i=1}^{4} C_{i} \rho_{i_{(\lambda)}},
$$

where $\rho_{1}, \ldots, \rho_{4}$ are four linearly independent solutions of the fourth-order eigenvalue equation

$$
\square^{2} \varepsilon_{(\lambda)}=\lambda \varepsilon_{(\lambda)} .
$$


We therefore find that, when the conformally invariant gauge functionals (10) are used, the admissible boundary conditions differ substantially from the magnetic and electric schemes outlined in Eqs. (1)-(3) and (4)-(6), and are conformally invariant by construction (with the exception of Eq. (15)).

Had we set to zero at the boundary $A_{k}(k=1,2,3)$ and the functional (10), we would not have obtained enough boundary conditions for ghost perturbations, since both choices lead to Dirichlet conditions on the ghost. The boundary conditions (13) are also very important since they ensure the vanishing of all boundary terms resulting from integration by parts in the Faddeev-Popov action. In the particular case when the 3 -surface $\Sigma_{1}$ shrinks to a point, which is relevant for (one-loop) quantum cosmology [5], the boundary conditions read (here $\Sigma$ is the bounding 3 -surface)

$$
\begin{gathered}
{\left[A_{b}\right]_{\Sigma}=0 \forall b=0,1,2,3} \\
{[\varepsilon]_{\Sigma}=0} \\
{[\partial \varepsilon / \partial \tau]_{\Sigma}=0}
\end{gathered}
$$

jointly with regularity at $\tau=0$ of $A_{b}, \varepsilon$ and $\frac{\partial \varepsilon}{\partial \tau}$. Many fascinating problems are now in sight. They are as follows:

(i) To prove uniqueness of the solution of the classical boundary-value problem

$$
\begin{gathered}
\square^{2} f=0 \\
{[f]_{\Sigma_{1}}=[f]_{\Sigma_{2}}=0,} \\
{[\partial f / \partial \tau]_{\Sigma_{1}}=[\partial f / \partial \tau]_{\Sigma_{2}}=0 .}
\end{gathered}
$$

(ii) To study the quantum theory resulting from the operator (11) for finite values of $\alpha$. Interestingly, the Feynman choice $\alpha=1$ does not get rid of the sixth-order nature of the operator $\mathcal{P}^{b c}$. 


\section{Quantized Maxwell Theory in a Conformally Invariant Gauge}

(iii) To evaluate the one-loop semiclassical approximation, at least when $\mathcal{P}^{b c}$ reduces to the form (12) in the presence of 3 -sphere boundaries. The ghost operator is then found to take the form

$$
\begin{aligned}
\square^{2} & =\frac{\partial^{4}}{\partial \tau^{4}}+\frac{6}{\tau} \frac{\partial^{3}}{\partial \tau^{3}}+\frac{3}{\tau^{2}} \frac{\partial^{2}}{\partial \tau^{2}}-\frac{3}{\tau^{3}} \frac{\partial}{\partial \tau} \\
& +\frac{2}{\tau^{2}}\left(\frac{\partial^{2}}{\partial \tau^{2}}+\frac{1}{\tau} \frac{\partial}{\partial \tau}\right)_{\mid i}{ }^{\mid i}+\frac{1}{\tau^{4}}\left({ }_{\mid i}^{\mid i}\right)^{2} .
\end{aligned}
$$

With a standard notation, we denote by $\mid$ the operation of covariant differentiation tangentially with respect to the 3 -dimensional Levi-Civita connection of the boundary. If one expands the ghost perturbations on a family of 3 -spheres centred on the origin as [1]

$$
\varepsilon(x, \tau)=\sum_{n=1}^{\infty} \varepsilon_{n}(\tau) Q^{(n)}(x),
$$

the operator (26), jointly with the properties of scalar harmonics, leads to the eigenvalue equation (cf. Eq. (19))

$$
\begin{aligned}
& \frac{d^{4} \varepsilon_{n}}{d \tau^{4}}+\frac{6}{\tau} \frac{d^{3} \varepsilon_{n}}{d \tau^{3}}-\frac{\left(2 n^{2}-5\right)}{\tau^{2}} \frac{d^{2} \varepsilon_{n}}{d \tau^{2}}-\frac{\left(2 n^{2}+1\right)}{\tau^{3}} \frac{d \varepsilon_{n}}{d \tau} \\
& +\left(\frac{\left(n^{2}-1\right)^{2}}{\tau^{4}}-\lambda_{n}\right) \varepsilon_{n}=0 .
\end{aligned}
$$

This equation admits a power series solution in the form

$$
\varepsilon_{n}(\tau)=\tau^{\rho} \sum_{k=0}^{\infty} b_{n, k}\left(n, k, \lambda_{n}\right) \tau^{k} .
$$

The values of $\rho$ are found by solving the fourth-order algebraic equation

$$
\rho^{4}-2\left(n^{2}+1\right) \rho^{2}+\left(n^{2}-1\right)^{2}=0,
$$

which has the four real roots $\pm(n \pm 1)$. Moreover, the only non-vanishing $b_{n, k}$ coefficients are of the form $b_{n, 4 k}, \forall k=0,1,2, \ldots$, and are given by (assuming that $b_{n, 0}$ has been fixed)

$$
b_{n, l}=\frac{\lambda_{n} b_{n, l-4}}{F(l, n, \rho)}, \forall l=4,8,12, \ldots,
$$


where we have defined $(\forall k=0,1,2, \ldots)$

$$
\begin{aligned}
F(k, n, \rho) & \equiv(\rho+k)(\rho+k-1)(\rho+k-2)(\rho+k-3)+6(\rho+k)(\rho+k-1)(\rho+k-2) \\
& -\left(2 n^{2}-5\right)(\rho+k)(\rho+k-1)-\left(2 n^{2}+1\right)(\rho+k)+\left(n^{2}-1\right)^{2}
\end{aligned}
$$

As far as we can see, the solution (28) can be expressed in terms of Bessel functions and of a new set of special functions (cf. Sec. 3.5 of Ref. [10]).

(iv) To include the effects of curvature. As shown in Ref. [8], if the background 4-geometry is curved, with Riemann tensor $R_{b c d}^{a}$, the conformally invariant gauge-averaging functional reads (cf. Eq. (10))

$$
\Phi(A) \equiv \square \nabla^{b} A_{b}+\nabla_{c}\left[\left(-2 R^{b c}+\frac{2}{3} R g^{b c}\right) A_{b}\right]
$$

It would be interesting to study the (one-loop) quantum theory, at least when $\alpha \rightarrow \infty$, on curved backgrounds like $S^{4}$, which is relevant for inflation [5], or $S^{2} \times S^{2}$, which is relevant for the bubbles picture in Euclidean quantum gravity, as proposed in Ref. [11].

To our knowledge, the form (11) of the differential operator on perturbations of the electromagnetic potential in the quantum theory, the boundary conditions (13)-(15), and the analytic solution (28)-(31) for ghost basis functions are entirely new. Thus, quantization via path integrals in conformally invariant gauges possesses some new peculiar properties, which are now under investigation for the first time. This, in turn, seems to add evidence in favour of Euclidean quantum gravity having a deep influence on current developments in quantum field theory [5].

The author is indebted to A. Yu. Kamenshchik and G. Pollifrone for scientific collaboration on Euclidean Maxwell theory and Euclidean quantum gravity over many years.

\section{References}

[1] G. Esposito, Class. Quantum Grav. 11 (1994) 905. 
[2] G. Esposito and A.Yu. Kamenshchik, Phys. Lett. B 336 (1994) 324.

[3] G. Esposito, A.Yu. Kamenshchik, I.V. Mishakov and G. Pollifrone, Class. Quantum Grav. 11 (1994) 2939.

[4] G. Esposito, A.Yu. Kamenshchik, I.V. Mishakov and G. Pollifrone, Phys. Rev. D 52 (1995) 2183.

[5] G. Esposito, A.Yu. Kamenshchik and G. Pollifrone, Euclidean Quantum Gravity on Manifolds with Boundary (Kluwer, Dordrecht, 1997).

[6] D.V. Vassilevich, J. Math. Phys. 36 (1995) 3174.

[7] D.V. Vassilevich, Phys. Rev. D 52 (1995) 999.

[8] M. Eastwood and M. Singer, Phys. Lett. 107 A (1985) 73.

[9] B.S. DeWitt, in Quantum Gravity 2, a Second Oxford Symposium, eds. C.J. Isham, R. Penrose and D.W. Sciama (Oxford, Clarendon Press, 1981).

[10] W. Magnus, F. Oberhettinger, and R. P. Soni, Formulas and Theorems for the Special Functions of Mathematical Physics (Springer-Verlag, Berlin, 1966).

[11] S.W. Hawking, Phys. Rev. D 53 (1996) 3099. 